\title{
Near infrared spectroscopy of the HADS stars V703 Sco and 1 Mon: First steps towards the use of near-IR spectroscopy for the study of $\delta$ Scuti stars
}

\author{
P.J. Amado ${ }^{1,2}$ \\ ${ }^{1}$ Instituto de Astrofísica de Andalucía (CSIC), Granada, Spain \\ ${ }^{2}$ Universidad de Granada, Granada, Spain
}

\begin{abstract}
Intermediate results from an on-going programme to study the possibilities of near-infrared spectroscopy are presented for the always difficult task of providing information on the mode identification of pulsating stars. High-resolution spectroscopy taken with CRIRES shows, for the first time, stellar absorption lines in the near-IR varying with the pulsation cycle in the high amplitude $\delta$ Scuti star 1 Mon. ${ }^{1}$
\end{abstract}

\section{Introduction}

Near-infrared, high-resolution spectrographs will be readily available at medium to large telescopes in the next decade. Existing instruments like PHOENIX and CRIRES, and next generation instruments like, for instance, NAHUAL (Martín et al., 2004), are possible due in part to the improvement in performance of near-IR detectors, now available in larger formats and with lower readout noise.

This region of the spectrum has rarely been used in the study of mainsequence classical pulsators, probably due to the lower flux emitted at these wavelengths by hot stars compared with that in the optical. This, together with the high temporal resolution and high $\mathrm{S} / \mathrm{N}$ needed for the study of pulsations, had become a strong impediment for the use of these type of data. With the advent of new, more efficient spectrographs at large telescopes, this difficulty can be surmounted and this methodology included in the battery of techniques

\footnotetext{
${ }^{1}$ Based on observations collected at the European Southern Observatory at La Silla and Paranal, Chile (CRIRES science verification program 60.A-9086)
} 
used in asteroseismology. This method will thus provide a large wavelength baseline for modal identification, which greatly improves the significance of the identification, as shown by Balona et al. (2001) for the optical $V$ and $I$ wavebands, when applying the photometric mode identification technique (e.g., Garrido, 2000). In this and future analyses, the same principle will be applied to spectroscopic data, and the first steps will be given towards the use of near-IR, high-resolution spectroscopy for the study of main sequence pulsating stars.

The main aim of this investigation is the detection of pulsations in the radial velocity (RV) curve or in the measurements of the equivalent width (EW) of the hydrogen lines in the low-resolution data provided by Sofl, and in the variations of the line profiles in the high-resolution data provided by CRIRES. An attempt to provide physical parameters of the stars from near-IR spectroscopy alone has also been carried out for V703 Sco (Amado et al., 2007) and is being attempted for 1 Mon.

\section{V703 Sco and 1 Mon}

Our target stars are High Amplitude Delta Scuti (HADS) pulsators, which are defined as $\delta$ Sct stars with a total amplitude in their visual light curves greater than 0.3 mag. This sample was selected for this study because of their high amplitude, their visibility and the number of periodicities already detected in their light curves.

The star V703 Sco (HD 160589) is a HADS with periods of $P_{1}=0.14996 \mathrm{~d}$ $\left(\nu_{1}=6.66844 \mathrm{~d}^{-1}\right), P_{2}=0.11522 \mathrm{~d}\left(\nu_{2}=6.67905 \mathrm{~d}^{-1}\right)$, which might be due to the fundamental and first overtone modes, with a ratio of 0.768 , and $P_{3}=0.09354 \mathrm{~d}\left(\nu_{3}=10.69061 \mathrm{~d}^{-1}\right)$ (Ponsen, 1961; Oosterhoff, 1966; Koen, 2001). The rotational velocity is $v \sin i=16 \mathrm{~km} \mathrm{~s}^{-1}$ and the spectral type is A9V (Houk, 1982).

The star 1 Mon (HD 40535) is also a HADS with periods $P_{1}=0.136 \mathrm{~d}$ $\left(\nu_{1}=7.346 \mathrm{~d}^{-1} ; \ell=0\right), P_{2}=0.134 \mathrm{~d}\left(\nu_{2}=7.475 \mathrm{~d}^{-1}\right)$ and $P_{3}=0.139 \mathrm{~d}$ $\left(\nu_{3}=7.217 \mathrm{~d}^{-1}\right)$. The last two periods belong to two $\ell=1$ modes, as detected by Balona \& Stobie (1980) and as identified by Balona et al. (2001) and Dupret et al. (2005). A fourth period $P_{4}=0.149 \mathrm{~d}\left(\nu_{4}=6.717 \mathrm{~d}^{-1}\right)$ was detected by Breger \& Kolenberg (2006). The star has also a low projected rotational velocity $\left(v \sin i=18.8 \pm 1.5 \mathrm{~km} \mathrm{~s}^{-1}\right.$; Solano \& Fernley, 1997) and a spectral type F2V (Rodríguez et al., 1994).

\section{Observations}

V703 Sco was observed during two nights, on the 12th and the 14th of September 2005, with Sofl on the NTT at La Silla, ESO. On each of these nights, 
the star was observed in a consecutive sequence of 6 exposures in the mode "AutoNodOnSlit", each one taken by nodding the star 60 arcsec on the slit. Exposure times were computed taking the average of 30 (NDIT) 12s (DIT) exposures with 3 integrations in position $A(N I N T=3)$ and another 3 in position $B$. The object frames were taken through a 0.6 arcsec slit to get the maximum possible resolution $(R \sim 1500)$. The data were reduced with IRAF (Tody, 1986).

1 Mon was observed simultaneously in near-IR spectroscopy and in Strömgren photometry at the beginning of March 2007. The photometric data were continuously acquired from the 27th of February to the 3rd of March at the Observatorio de Sierra Nevada (OSN; Granada, Spain), except for the very same night when the star was observed by CRIRES. The spectroscopic observations were carried out with CRIRES at the VLT in Science Verification time during the night of March the 2 nd. The target was monitored continuously during $\approx 3.5$ hours to cover one pulsation cycle. Observations were performed at three different settings with central wavelengths $1643.9,1693.8$ and $1732.7 \mathrm{~nm}$. That configuration of the observations provided 12 pieces of the $H$-band spectrum starting at 1617 and ending at $1746 \mathrm{~nm}$, covering, therefore, a total range in wavelength of $\sim 100 \mathrm{~nm}$ (see Fig. 1, top panel). A slit width of 0.4 arcsec was used to obtain a resolution of $\sim 35000$ measured from the FWHM of several isolated telluric absorption lines.

\section{Correction of the telluric spectrum}

Ground-based, near-IR spectra show, even within the well established photometric $J, H$ and $K$ bands, absorption features due to the Earth's atmosphere. Classical methods for correcting the data from these variable absorption features are difficult to apply to observations based in the rapid and continuous acquisition of time series spectra. Observations of telluric standards are thus not that useful, as these time series take longer than the time scale of the atmospheric variations (of the order of a few to tens of minutes), and take the target to different airmasses than those at which the standars were observed.

The data presented in this paper, especially the low-resolution data, were acquired in order to check for the effect of not properly resolving the variable atmospheric absorption lines (intrinsically narrow $\sim 5 \mathrm{~km} \mathrm{~s}^{-1}$ ). The correction of the atmospheric absorption spectrum in low-resolution data is complicated by the enormous amount of blended lines and bands at this resolution, even if the $H$ band is affected the least compared with the $J$ or $K$ bands. In Fig. 1, an $H$-band synthetic spectrum with the physical parameters of V703 Sco is shown, together with a telluric spectrum of the same region at the resolution given by Sofl (top, $\sim 1500$ ) and CRIRES (bottom, $\sim 35000$ ). It can be seen that the 
regions free of telluric absorption are very small in the low-resolution data and, even then, they coincide with regions of stellar absorption, such as the end of the hydrogen Brackett series or some of the broad H I Brackett lines. On the other hand, it can be seen that, at higher spectral resolution, it becomes easier to identify the sections of the spectrum where spectral information or stability is affected due to strong, saturated or variable telluric lines (for studies of these effects see, for instance, Vacca et al., 2003; Pontoppidan \& van Dishoeck, 2004; Bailey et al., 2007). In principle, the effect in the normalisation of low-resolution spectra from lack of good continuum regions, due to the blending of the wings of the broad hydrogen lines with the telluric absorption features, should affect the measurements of the EW of the line.

\section{Physical parameters from near-IR spectroscopy}

For V703 Sco, synthetic spectra were computed from Kurucz's model atmosphere grids, degraded to the resolution of the data and compared with the overall mean of the whole set of spectra. The best fit to the data is obtained for a model with $[\mathrm{Fe} / \mathrm{H}]=-0.5,6250<T_{\text {eff }}<6750 \mathrm{~K}$ and a $\log g=4.5$ (Amado et al., 2007) implying that V703 Sco is a SX Phe star. However, the high gravity derived from the synthetic spectra contradicts what would be expected from the periods of this star, which, interpreted as low radial order frequencies, imply an evolved status. Furthermore, the photometric indices and the space motions point towards a normal Pop. I HADS classification (Rodríguez, private communication). Therefore, some doubts still exist on the parameter determination for this star, even more taking into account that the LTE modelling of the near-IR hydrogen lines might fail to reproduce the strength of the lines (Przybilla \& Butler, 2004).

Synthetic spectra will also be computed for 1 Mon and fitted to the observed metallic lines in order to determine the physical parameters, providing, for the first time, a metallicity determination for this star.

\section{Effect of pulsation in the near-IR}

As mentioned above, the idea behind observing HADS stars was to assure an amplitude of pulsation large enough to be easily recovered in our data at both low and high resolution. Below, some preliminary results are provided.

\section{Low resolution data}

HADS objects show pulsation cycles with variations in $T_{\text {eff }}$ of the order of 1000 $\mathrm{K}$ and in $\log g$ of the order of 0.35 dex (Rolland et al., 1991; Rodríguez et al., 

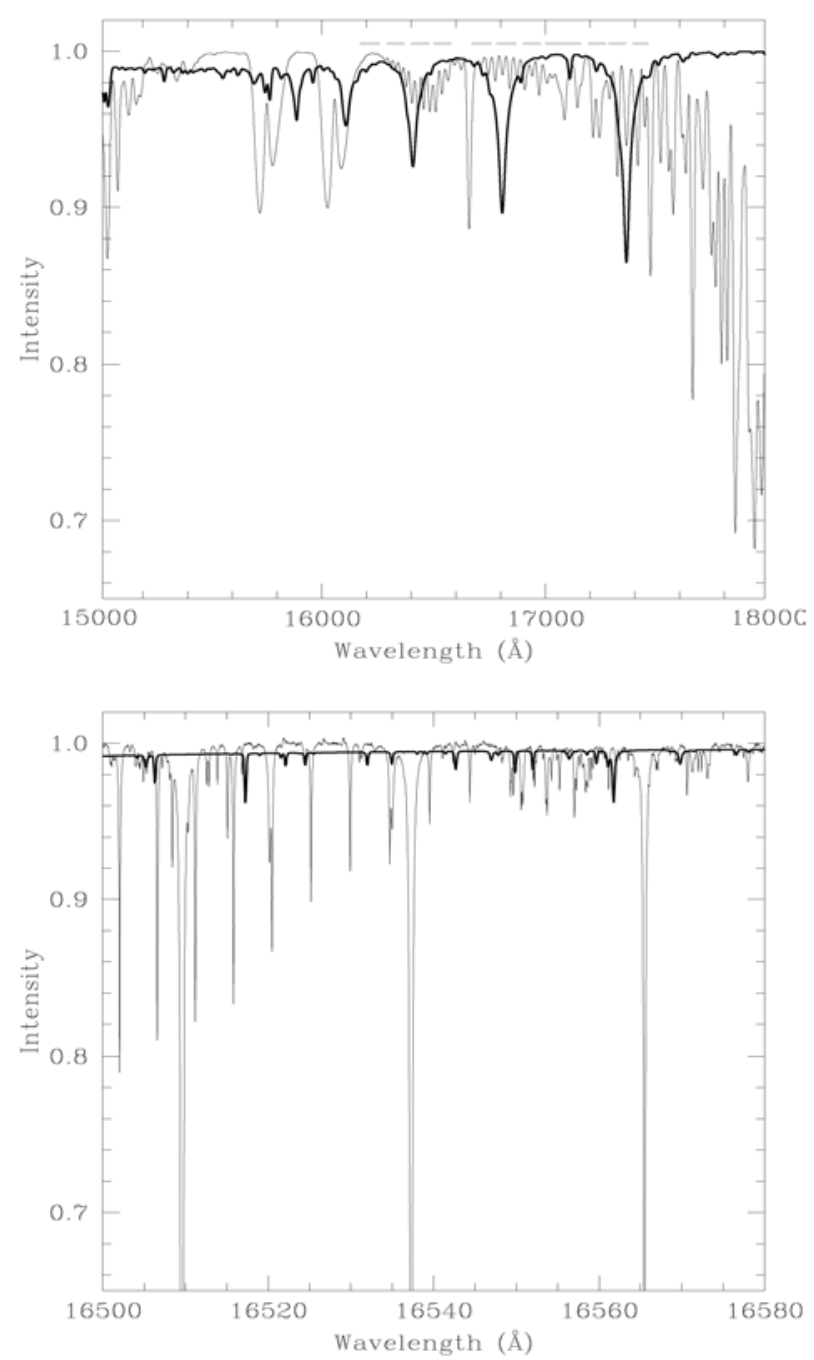

Figure 1: top panel: $H$-band synthetic spectrum computed with the physical parameters of V703 Sco (thick line) together with a telluric spectrum of the same region (thin line) at the resolution given by Sofl $(\sim 1500)$. Bottom panel: the same as for the top panel but at the resolution provided by CRIRES $(\sim 35000)$. The wavelength region of this spectrum corresponds to one of the thin lines, delimiting the wavelength regions of our CRIRES data, plotted above the continuum in the top panel. 


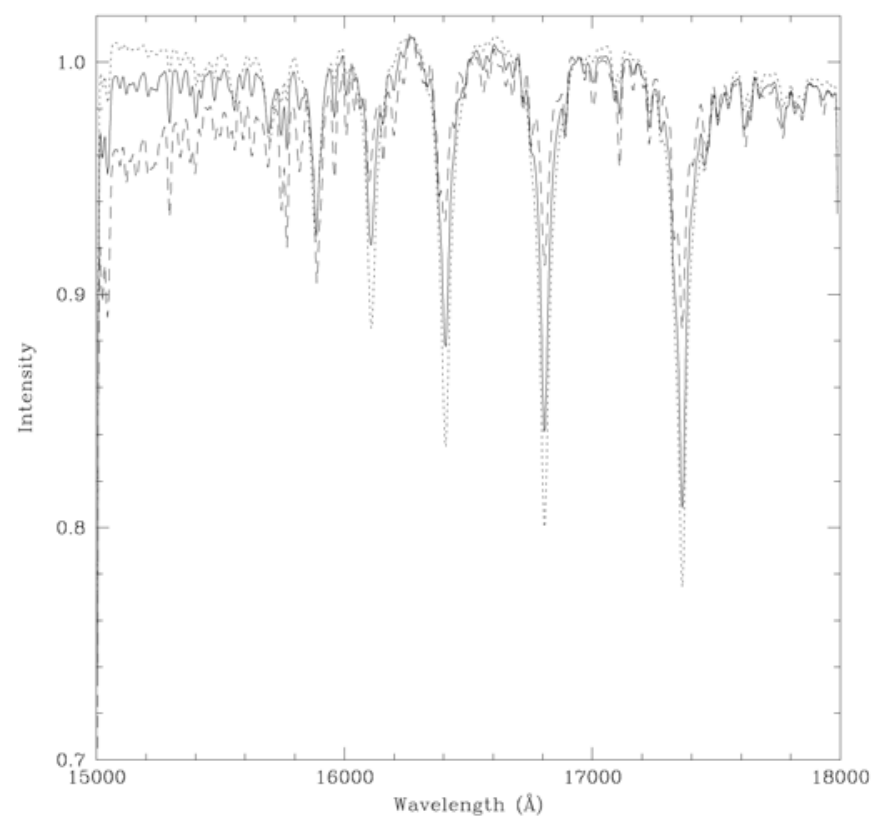

Figure 2: Normalised, synthetic stellar flux spectra for $T_{\text {eff }}=6000$ (dashed line), 7000 (solid line) and $8000 \mathrm{~K}$ (dotted line), $\log g=4.50$ and solar metallicity, in the near-IR region, at the resolution of Sofl $(\sim 1500)$.

1992). In Fig. 2, Kurucz's synthetic flux spectra with $T_{\text {eff }}=6000,7000$ and $8000 \mathrm{~K}, \log g=4.50$ and solar metallicity, are shown. These spectra were "normalised" by dividing them with a blackbody function of $T_{\mathrm{eff}}=7000 \mathrm{~K}$, which had been previously fitted to a continuum region of the spectra around $1700 \mathrm{~nm}$. This procedure was used with the observed low-resolution data of V703 Sco. It can be seen that the effect of increasing the temperature is basically a change in the depth of the hydrogen lines and in the shape of the spectral energy distribution (SED).

The dispersion in the RV and EW measurements in our data (taken at a high airmass) is too high to detect the pulsations. A small variation of the shape of the SED, of the same order as that mentioned above for the synthetic spectra, can be detected in the data. However, whereas the change in the depth of the hydrogen lines goes from $4 \%$ to $7 \%$ of the value of the continuum in the synthetic spectra, no change larger than around $1 \%$ is seen in $\mathrm{H}$ I (4-7) 


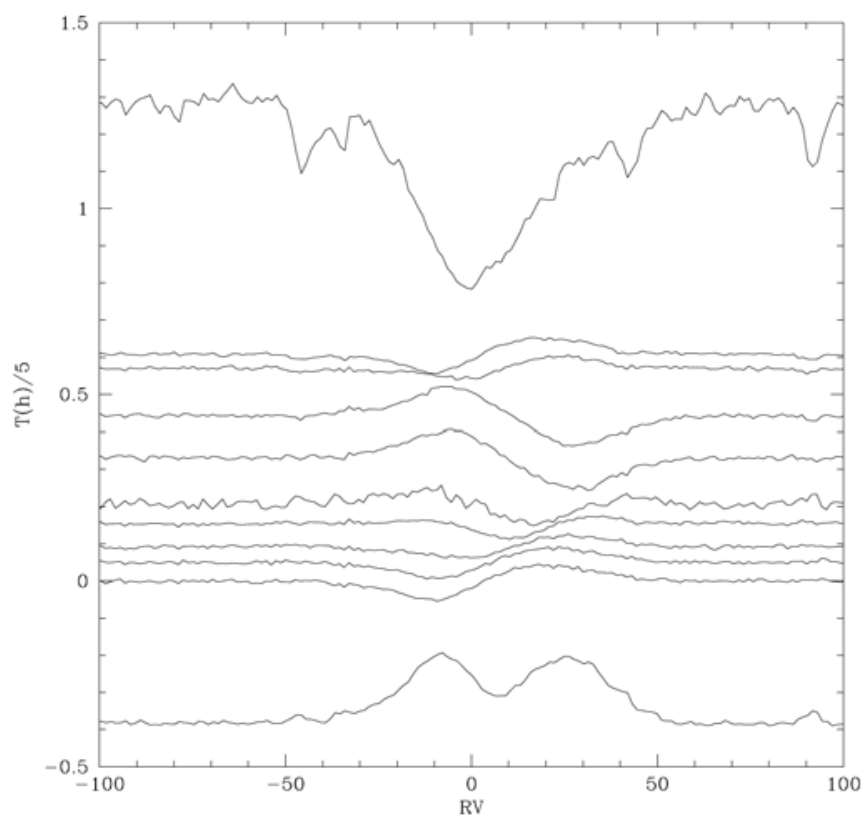

Figure 3: Standard deviation with respect to the mean (bottom), differences with respect to the mean profile time series, and mean profile (top) of the Si I $\lambda 1733.8551$ $\mathrm{nm}$ line, in the CRIRES spectrum of 1 Mon. The ordinate axis shows the elapsed time with respect to the time of acquisition of the first spectrum, in fifths of an hour.

in the observed spectra. In principle, a study of the pulsation cycle of V703 Sco would have been possible with these type of data if better quality spectra with a somewhat higher resolution had been used. Of course, a better temporal resolution is definitely needed.

High resolution data

The work on the CRIRES data is in progress but the preliminary results are encouraging.

In the $100 \mathrm{~nm}$ of the $H$-band spectrum covered by our observations, several lines are seen to vary in a standard deviation plot. Figure 3 shows one of these lines, corresponding to the Si I $\lambda 1733.8551 \mathrm{~nm}$, averaged over the whole time series (top), the differences of the individual spectra with respect to this average (centre) and the standard deviation from the average of the intensity at each 

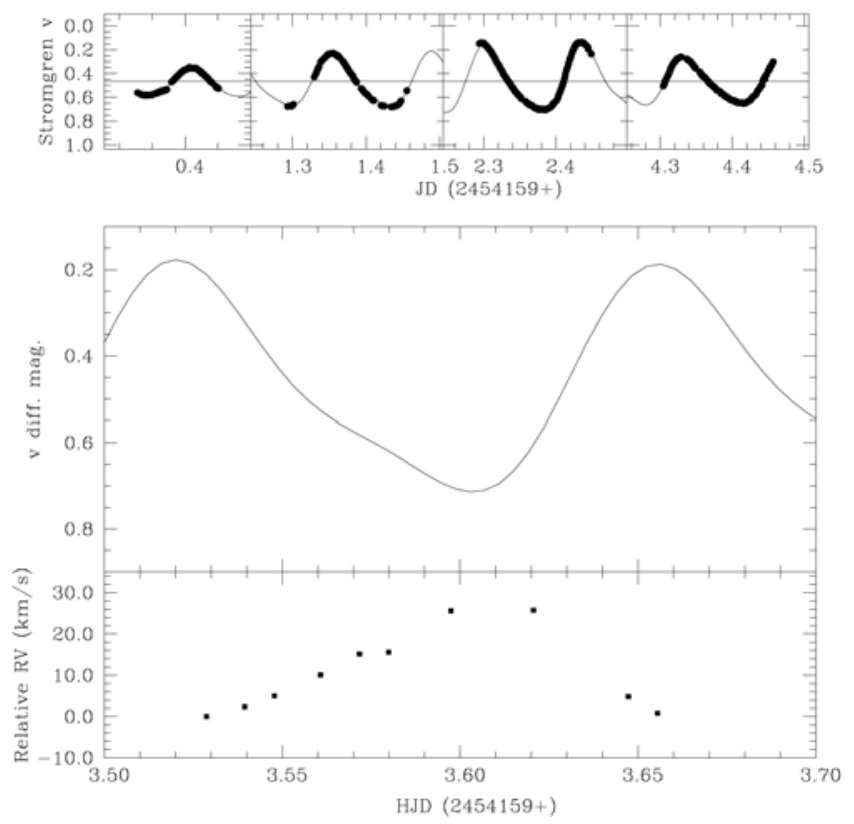

Figure 4: Top panel: Observed Strömgren $v$ differential light curve for the four nights of the observing run, together with a theoretical light curve determined from the main frequencies found in the data. Bottom panel: Zoom on one of the nights, showing the theoretical light curve (solid line; no photometric observations taken) and the radial velocities measured from the CRIRES spectra (square symbols).

pixel (bottom). Radial velocity measurements in this line are shown in Fig. 4, together with the Strömgren $v$ light curve collected at OSN. Unfortunately, no photometric data were acquired during the night of CRIRES observations and only an interpolation of the light curve computed with the detected frequencies can be provided. This preliminary RV curve appears in anti-phase with the light curve as expected, with an amplitude from maximum to minimum of 25.6 $\mathrm{km} \mathrm{s}^{-1}$, larger than those quoted by Smith $\left(1982,18 \mathrm{~km} \mathrm{~s}^{-1}\right)$ and Balona \& Stobie $\left(1980,15 \mathrm{~km} \mathrm{~s}^{-1}\right)$ probably because of beating effects. An in-depth analysis of this dataset is being conducted and will be the subject of a future work. 


\section{Conclusions and future work}

The results obtained for $\mathrm{V} 703 \mathrm{Sco}$ show that the low resolution provided by instruments like Sofl $(R \sim 1500)$ is not sufficient to produce RV curves of the required precision, or for the study of line-profile variations. Nevertheless, even at this resolution, it might be worth it to address the question of weather a study of EW time series might have been possible with higher quality data, resulting in precise enough curves from which to extract the amplitudes and phases of the modes, as done in the optical by Dall et al. (2003).

For the first time, variations in near-IR absorption lines due to pulsation have been detected and measured for a $\delta$ Scuti star. As demonstrated with CRIRES data, spectroscopic techniques already used in the optical (moments method, line profile fitting, Doppler imaging) can and will be applied to our data to extract information from this spectral region that can be used to constrain and help in the identification of the modes. The diagnostic potential of this region comes from the different sensitivity of the absorption lines to changes in $T_{\text {eff }}$ and $\log g$ with respect to the optical, and from the interplay between the pulsation and the limb-darkening effects. However, more theoretical work is needed, specially for the extension to this wavelength region of the well known amplitude-ratio and phase-difference plots used in modal discrimination.

In the near future, proposals to observe $\delta$ Scuti stars of various amplitudes with CRIRES and other near-IR spectrographs will be submitted. Morover, simultaneous high-resolution spectroscopy in the optical and in the near-IR will be acquired for these studies.

Acknowledgments. PJA acknowledges the staff of La Silla Paranal observatory for its helpfulness, specially Michael Sterzik and Maarten Baes for preparing and taking the observations of V703 Sco, and the Paranal staff for the observations of 1 Mon with CRIRES in Science Verification mode. The author also acknowledges financial support from a "Ramón y Cajal" contract of the Spanish Ministry of Education and Culture. He also thanks the help provided with the careful reading of the manuscript by J.C. Suárez and C. Rodríguez.

\section{References}

Amado, P. J., Suárez, J. C., Garrido, R., et al. 2007, CoAst 150, 311

Bailey, J., Simpson, A., \& Crisp, D. 2007, PASP 119, 228

Balona, L. A., Bartlett, B., Caldwell, J. A. R., et al. 2001, MNRAS 321, 239

Balona, L. A., \& Stobie, R. S. 1980, MNRAS 190, 931

Breger, M., \& Kolenberg, K., 2006, A\&A 460, 167 
Dall, T. H., Handler, G., Moalusi, M. B., \& Frandsen, S. 2003, A\&A 410, 983

Dupret, M.-A., Grigahcène, A., Garrido, R., et al. 2005, MNRAS 361, 476

Garrido R., 2000, in ASP Conf. Ser. 210: Delta Scuti and Related Stars

Photometric Modal Discrimination in $\delta$ Scuti and $\gamma$ Doradus Stars.p. 67

Houk N., 1982, in Michigan Spectral Survey, Ann Arbor, Dep. Astron., Univ.

Michigan, 3 (1982) Catalogue of two-dimensional spectral types for the HD stars,

Vol. 3. pp $0-+$

Koen, C. 2001, MNRAS 321, 44

Martín, E. L., Guenther, E. W., Caballero, J. A., et al. 2004, Astronomische Nachrichten Supplement 325, 132

Oosterhoff, P. T., 1966, Bull. Astron. Inst. Netherlands 18, 140

Ponsen, J. 1961, Bull. Astron. Inst. Netherlands 15, 325

Pontoppidan, K. M., \& van Dishoeck, E. F. 2004, astro-ph, 0405629

Przybilla, N., \& Butler, K. 2004, ApJ 609, 1181

Rodriguez, E., Lopez de Coca, P., Rolland, A., et al. 1994, A\&AS 106, 21

Rodriguez E., Rolland A., Lopez de Coca P., et al. 1992, A\&AS 93, 189

Rolland, A., Rodríguez, E., Lopez de Coca, P., \& Garcia-Pelayo, J. M. 1991, A\&AS 91, 347

Smith, M. A. 1982, ApJ 254, 242

Solano, E., \& Fernley, J. 1997, A\&AS 122, 131

Tody D., 1986, in Crawford D., ed., Instrumentation in Astronomy VI Vol. 627, The iraf data reduction and analysis system. p. 733

Vacca, W. D., Cushing, M. C., \& Rayner, J. T. 2003, PASP 115, 389 